\title{
Standards of Practice in Acute Ischemic Stroke Intervention: International Recommendations
}

Laurent Pierot ${ }^{1}$ Mahesh V Jayaraman ${ }^{2}$ Istvan Szikora ${ }^{3}$ Joshua A Hirsch ${ }^{4}$ Blaise Baxter ${ }^{5}$ Shigeru Miyachi ${ }^{6}$ Jeyaledchumy Mahadevan ${ }^{7}$ Winston Chong, ${ }^{8}$ Peter J Mitchell, ${ }^{9}$ Alan Coulthard, ${ }^{10}$ Howard A Rowley, ${ }^{11}$ Pina C Sanelli, ${ }^{12}$ Donatella Tampieri, ${ }^{13}$ Patrick A Brouwer, ${ }^{14}$ Jens Fiehler, ${ }^{15}$ Naci Kocer, ${ }^{16}$ Pedro Vilela, ${ }^{17}$ Alex Rovira, ${ }^{18}$ Urs Fischer, ${ }^{19}$ Valeria Caso, ${ }^{20}$ Bart van der Worp, ${ }^{21}$ Nobuyuki Sakai, ${ }^{22}$ Yuji Matsumaru, ${ }^{23}$ Shin-ichi Yoshimura, ${ }^{24}$ Rene Anxionnat, ${ }^{25}$ Hubert Desal, ${ }^{25}$ Luisa Biscoito, ${ }^{26}$ José Manuel Pumar, ${ }^{27}$ Orlando Diaz, ${ }^{28}$ Justin F Fraser, ${ }^{29}$ Italo Linfante, ${ }^{30}$ David S Liebeskind, ${ }^{31}$ Raul G Nogueira, ${ }^{32}$ Werner Hacke, ${ }^{33}$ Michael Brainin, ${ }^{34}$ Bernard Yan, ${ }^{35}$ Michael Soderman, ${ }^{14}$ Allan Taylor, ${ }^{36}$ Sirintara Pongpech, ${ }^{37}$ Michihiro Tanaka, ${ }^{38}$ Karel Terbrugge ${ }^{39}$ Asian-Australian Federation of Interventional and Therapeutic Neuroradiology (AAFITN), Australian and New Zealand Society of Neuroradiology (ANZSNR), American Society of Neuroradiology (ASNR), Canadian Society of Neuroradiology (CSNR), European Society of Minimally Invasive Neurological Therapy (ESMINT), European Society of Neuroradiology (ESNR), European Stroke Organization (ESO), Japanese Society for NeuroEndovascular Therapy (JSNET), the French Society of Neuroradiology (SFNR), Ibero-Latin American Society of Diagnostic and Therapeutic Neuroradiology (SILAN), Society of Neurolnterventional Surgery (SNIS), Society of Vascular and Interventional Neurology (SVIN), World Stroke Organization (WSO), World Federation of Interventional Neuroradiology (WFITN).

\section{PREAMBLE}

After the five positive randomized controlled trials showing the benefit of mechanical thrombectomy (MT) in the management of acute ischemic stroke (AIS) with emergent large vessel occlusion (ELVO), a multisociety meeting was organized during the 16th Congress of the World Federation of Interventional and Therapeutic Neuroradiology (WFITN), October 2015, Gold Coast (Australia). This meeting was dedicated to the training of physicians performing MT, and recommendations were published thereafter in multiple scientific journals. ${ }^{1}$

The same group of scientific societies decided to organize a similar meeting during the 17th WFITN Congress, October 2017, Budapest (Hungary). This multisociety meeting was dedicated to standards of practice in acute ischemic stroke intervention (AISI), aiming for a consensus on the minimum requirements for centers providing such treatment.

In an ideal situation, all patients would be treated at a center offering a full spectrum of neuroendovascular care (a level 1 center). However, for geographical reasons, some patients are unable to reach such a center in a reasonable period of time. With this in mind, the group paid special attention to define recommendations on the prerequisites of organizing stroke centers providing MT for AIS, but not for other neurovascular diseases (a level 2 center). Finally, some centers will have a stroke unit and offer intravenous thrombolysis, but not any endovascular stroke therapy (a level 3 center). Together, these level 1, 2, and 3 centers form a complete stroke system of care. The requirements for these centers are summarized in Table 1.

Due to the relatively short time elapsed since the evidence in favor of MT has been published, some organizational aspects still

This article was first published as: Pierot L, Jayaraman MV, Szikora I, et al. Standards of practice in acute ischemic stroke intervention: international recommendations. Journal of Neuro Interventional Surgery Published Online First: 28 August 2018; doi: 10.1136/neurintsurg-2018-014287 and is reprinted with permission. http://dx.doi.org/10.3174/ajnr.A5853 require scientific validation. However, considering the extremely fast growth of such activities around the world, the multisociety group considered it timely and rational to set-up recommendations and a framework for the development of MT services in all parts of the world. The requirements included in this document are proposed to help countries and centers to properly implement MT.

\section{COMPOSITION OF THE CONSENSUS GROUP}

This working group is composed of delegates from the following societies: Asian-Australian Federation of Interventional and Therapeutic Neuroradiology (AAFITN), Australian and New Zealand Society of Neuroradiology (ANZSNR), American Society of Neuroradiology (ASNR), Canadian Society of Neuroradiology (CSNR), European Society of Minimally Invasive Neurologic Therapy (ESMINT), European Society of Neuroradiology (ESNR), European Stroke Organization (ESO), Japanese Society for NeuroEndovascular Therapy (JSNET), Ibero-Latin American Society of Diagnostic and Therapeutic Neuroradiology (SILAN), Society of NeuroInterventional Surgery (SNIS), Society of Vascular and Interventional Neurology (SVIN), World Stroke Organization (WSO), World Federation of Interventional Neuroradiology (WFITN).

\section{DEFINITIONS}

Neuroendovascular procedures: minimally invasive, image guided procedures to treat diseases of the brain and spinal cord. These include embolization, for treatment of intracranial aneurysms, arteriovenous malformations, tumors, and revascularization techniques, such as angioplasty and stent placement for atherosclerotic disease.

Acute ischemic stroke intervention (AISI) involves percutaneous endovascular procedures to treat ischemic stroke in adults and children, and may involve thrombectomy, aspiration, percu- 
Table 1: General summary of capabilities of level 1,2 , and 3 centers

\begin{tabular}{|c|c|c|c|}
\hline & Level 1 Center & Level 2 Center & Level 3 Center \\
\hline $\begin{array}{l}\text { Offers full spectrum of } \\
\text { neuroendovascular therapy } \\
\text { (including aneurysm treatment, } \\
\text { surgical and endovascular, } \\
\text { arteriovenous malformations, a } \\
\text { rteriovenous fistulas, etc) }\end{array}$ & Yes & No & No \\
\hline Offers endovascular stroke therapy & Yes & Yes & No \\
\hline $\begin{array}{l}\text { Offers intravenous tissue } \\
\text { plasiminogen activator }\end{array}$ & Yes & Yes & Yes \\
\hline $\begin{array}{l}\text { Minimum No of stroke patients per } \\
\text { year }\end{array}$ & 250 & 100 & 50 \\
\hline $\begin{array}{l}\text { Minimum thrombectomy volume } \\
\text { per year }\end{array}$ & 50 & 50 & N/A \\
\hline Dedicated neuro-intensive care unit & Yes & Optional & Not needed \\
\hline Dedicated stroke unit & Yes & Yes & Yes \\
\hline Open neurosurgical services on site & Yes & Optional & Not needed \\
\hline Geographic restriction? & No & $\begin{array}{l}\text { Yes (should be more than } 2 \text { hours' transport } \\
\text { time from a level } 1 \text { center) }\end{array}$ & No \\
\hline Inter-facility transfers & $\begin{array}{l}\text { Receives cases } \\
\text { from level } 1 \\
\text { and } 2 \\
\text { centers }\end{array}$ & $\begin{array}{l}\text { Will transfer some cases to a level } 1 \text { center. } \\
\text { Will occasionally receive transfers from } \\
\text { level } 3 \text { centers if no level } 1 \text { center is } \\
\text { available within } 2 \text { hours from the level } 3 \\
\text { center }\end{array}$ & $\begin{array}{l}\text { Has standardized transfer processes } \\
\text { in place with a level } 1 \text { center } \\
\text { (preferable), or a level } 2 \text { center }\end{array}$ \\
\hline
\end{tabular}

taneous transluminal angioplasty, and stent implantation, as well as superselective drug infusion.

Stroke unit: a dedicated, geographically clearly defined area or ward in a hospital where stroke patients are admitted and cared for by a multi-professional team (medical, nursing, and therapy staff) who have specialist knowledge, training, and skills in stroke care with well defined individual tasks, regular interaction with other disciplines, and stroke leadership. This team shall coordinate stroke care through regular (weekly) multi-professional meetings (http://stroke.ahajournals.org/content/44/3/828\#T1).

Stroke center: a hospital infrastructure and related processes of care that provide the full pathway of stroke unit care. A stroke center is the coordinating body of the entire chain of care. This covers prehospital care, emergency room assessment and diagnosis, emergency medical treatment, stroke unit care, ongoing rehabilitation, and secondary prevention, and access to related neurosurgical and vascular intervention. A stroke unit is the most important component of a stroke center. A stroke center provides stroke unit services for the population of its own catchment area and serves as a referral center for peripheral hospitals with stroke units in case their patients need services that are not locally available (http://stroke.ahajournals.org/content/44/3/828\#T1).

\section{BACKGROUND AND SIGNIFICANCE}

AIS caused by ELVO is the leading cause of adult disability in the world. ${ }^{2}$ Strokes caused by occlusion of the large intracranial vessels, such as the internal carotid artery, proximal middle cerebral artery, or basilar artery have low rates of response to intravenous tissue plasminogen activator and, subsequently, poor outcomes. ${ }^{3}$ The major revolution in acute stroke intervention began in 2015 when five randomized trials showed that rapid MT significantly improves outcomes in anterior circulation (internal carotid artery, M1) ELVO stroke patients. ${ }^{4-8}$ The degree of benefit is profound, with a number needed to treat as low as 2.5 to have one patient be less disabled. ${ }^{9,}{ }^{10}$ Few, if any, therapies in medicine can approach that level of benefit. Two additional trials have further confirmed that indeed rapid thrombectomy dramatically improves outcomes, including up to 24 hours from the last known normal. ${ }^{11-14}$

Training guidelines for physicians performing AISI were already proposed by the same working group. ${ }^{1}$ Delivering the benefit of this therapy to a population that is applicable in diverse localities throughout the world, as reflected by the breath of international societies sponsoring this guideline, requires a concerted effort. Critical to this is ensuring the proper facility capabilities to deliver this treatment in a safe yet timely fashion.

The goal of this document is to provide recommendations that outline the minimum requirements to provide AISI to as large of a population as possible, including those that do not have timely access to a level 1 center, which is capable of treating all vascular diseases of the brain and spine.

\section{PURPOSE}

This is a document which provides recommendations based on expert opinions and best available evidence, in relation to the optimal conditions for the safe practice of AISI.

In order to replicate the dramatic results of the major randomized trials, we must ensure patients throughout the world are treated in a center with the capabilities necessary to handle not just the procedural aspects, but also the medical management of the patient prior to, during, and post-thrombectomy.

These general recommendations are not a substitute for existing national and regional guidelines, recommendations, and regulations in the field of AIS. Rather, this describes the minimum organization and workload that, based on expert consensus, is necessary for a hospital to practice AISI.

The best option for the management of AIS is to have patients transferred to and treated in high volume, level 1 centers, as demonstrated by scientific evidence. ${ }^{15}$ However, in some situations, specifically due to geographical, traffic, and transportation con- 
ditions, access of patients to such centers in an acceptable time frame may not be possible. In that case, it would be wise to have a system of care that incorporates level 2 centers, able to provide AISI but not necessarily the full spectrum of neuroendovascular procedures.

\section{WHERE IS AISI PERFORMED?}

The practice of AISI should ideally take place in healthcare institutions that routinely provide services for all neurological disorders and neurointerventional treatments to patients with all kinds of neurovascular disorders (level 1 centers). Recommendation for these centers have been recently published. ${ }^{16}$

However, if a level 1 center is not regionally available, a center treating only ischemic stroke (level 2) can be established under the following conditions:

- There is no level 1 center available within 2 hours of interfacility transport time.

- The level 2 center must care for a reasonable number of AIS treatments a year (at least 100 treatments, including intravenous thrombolysis and AISI).

- The institution must incorporate an acute stroke center or stroke unit with fully trained stroke physicians.

- It is highly recommended that the level 2 center is organized in cooperation with a level 1 center, and should pursue the objective of collaborative work with the level 1 center for neurointervention training, continuous medical education, mortality and morbidity rounds, expertise advice by tele-consultations or by practice, 24 hour/7 week day coverage, referrals, among other).

\section{LEVEL 2 CENTER: STANDARDS OF PRACTICE}

For those centers established under these conditions the standards of practice described below apply.

\section{Facilities}

Facilities that must be available on site include:

- Stroke unit beds: a sufficient number of stroke unit beds should be available in stroke units to accommodate interventionally treated stroke patients at any time.

- Intensive care unit.

- A radiology/neuroradiology service, with competence in neuroimaging, and a suitable angiography room (as defined below): high quality, rapidly available noninvasive imaging is vital to the management of the acute stroke patient. At a minimum, CT scanners should be available on a $24 / 7$ basis to image patients with noncontrast CT and CT angiography. The availability of CT perfusion and/or MRI may also assist in patient selection for AISI beyond 6 hours from onset. The necessary technologists and support personnel for this imaging should be available and onsite at the time of patient admission. Diagnostic radiologists/neuroradiologists with sufficient training and experience in the interpretation of these imaging studies shall be available on a $24 / 7$ basis. Finally, cerebrovascular ultrasound facilities will be available.

- A team of trained acute stroke neurointerventionists.
- A dedicated 'stroke unit' and a 'stroke team' with fully trained stroke physicians.

- A department of neurosurgery ideally in house or, if that is not possible, in a nearby hospital.

\section{Angiography Suite}

A suitable interventional angiographic suite implies the ability to routinely accommodate general anesthesia. Optimally, procedures should be carried out under the image guidance of a biplane digital angiography unit with flat panel CT capabilities and necessary software and hardware to perform high quality cerebral angiography.

As a minimum, each suite should include a single plane high resolution digital subtraction angiography unit with road mapping capabilities.

Radiation protection measures in accordance with national regulations should be in place with designated individuals responsible for carrying out the necessary checks and audits.

\section{Treatment Availability}

AISI should be offered to every appropriate patient according to international guidelines, not excluding/discriminating against any patient, appropriate at the right time to obtain the best results, with population treatment access equity, in centers providing safe, effective, and efficient treatment.

A suitable level 2 center should be able to provide the services defined in the definition section, on a full time basis, $24 / 7$, all year around.

\section{Procedural Volume}

The randomized trials demonstrating a clear benefit from thrombectomy were almost exclusively performed in high volume centers. It has been shown that high volume centers have a significantly lower mortality, even if the patient has to be transferred from a low volume center. Rinaldo et al found that centers performing 35 or more thrombectomy cases per year would classify as 'high volume' and offer the lowest mortality rate for patients. ${ }^{15}$ Similarly, the American College of Cardiology Foundation, the American Heart Association, and the Society for Cardiovascular Angiography and Interventions suggest a minimum of 36 percutaneous coronary interventions for acute myocardial infarction per year per center as a minimum requirement. ${ }^{17}$

We acknowledge that the thresholds listed below are generally low. Multiple regional/national recommendations with higher limits are available and should be observed in regions/countries having already advanced healthcare networks providing services for AIS patients. The current recommendations are international and have to be compatible with the development of this new activity in areas and countries where there had been previously limited availability. Subsequently, these thresholds should be considered as the minimum caseload providing the lowest limit of safe operation. With the increased implementation of AISI in the world, it may be desirable to revise these thresholds in the future.

On the other hand, we also acknowledge that these thresholds are potentially difficult to reach in newly created level 2 centers and recognize that, during a transitory period, the activity can be 
below the threshold numbers, as long as it is expected that the volumes would be reached within 12-24 months.

With all of the above in mind, the suggested thresholds for annual procedure volume to maintain the competence for AIS endovascular treatment are the following:

- Each level 2 center shall perform a minimum number of intracranial thrombectomy procedures for ELVO per year. The global consensus group recommends a minimum of 50 procedures per center per year.

- Including the aforementioned thrombectomy procedures, each level 2 center shall perform a minimum total number of neuroendovascular procedures (diagnostic and interventional) per year according to national requirements. The global consensus group recommends a minimum of 120 per center per year.

- Each neurointerventionist working in a level 2 center must perform a minimum number of acute intracranial thrombectomy procedures per year, in accordance with national requirements. The global consensus group recommends a minimum of 15 procedures per neurointerventionist per year.

- In addition to the aforementioned thrombectomy procedures, each neurointerventionist in a level 2 center should perform a minimum number of total neuroendovascular procedures per year according to national requirements. The global consensus group recommends a minimum of 50 procedures per neurointerventionist per year.

\section{Operational Guidelines/Medical Personnel}

\subsection{Stroke Team}

Outstanding stroke care does not exist in a vacuum solely focused on the procedure but instead is part of a successful multidisciplinary team. The stroke team comprises fully trained stroke physicians (vascular neurologists or neurointensivists), allied professionals, and nurse that are all led by a stroke physician with a strong background in the management of neurovascular disease.

\subsection{Level 2 Stroke Intervention Team}

- The team should have a minimum of three clinicians with training and qualification in AISI. ${ }^{18}$

- The team should organize 24/7/365 acute ELVO stroke coverage (possibly in a rotation system organized with other level 2 centers or a level 1 center).

- It is recommended that stroke neurointerventionists involved in AISI maintain outpatient clinics for follow-up and have admitting privileges either in units/beds dedicated to Interventional neuroradiology or in other appropriate inpatient facilities.

- The stroke neurointerventionist/interventionist, in collaboration with the stroke team, should have shared responsibility for preoperative and postoperative patient care with input from the appropriate specialties.

- AISI should ideally be practiced in neurointerventional teams with the possibility to exchange experience and knowledge. Clinical research should be encouraged. The solitary practice of AISI is strongly discouraged.

\subsection{Anesthesia Team}

There shall be 24/7 in hospital anesthesia coverage with anesthetists with experience in caring for patients undergoing AISI. At many centers, the use of anesthesia, whether monitored anesthetic care or general anesthesia, is routine during thrombectomy. Even at centers primarily using moderate sedation, patients may deteriorate clinically prior to, or during, the procedure such that immediate access to general anesthesia is necessary to safely complete the procedure.

\subsection{Others}

Given the significant amount of assistance stroke patients need re-integrating into the community, the center should have access to physical therapy, speech therapy, occupational therapy services, as well as a coordinated plan for assessment for rehabilitation needs.

\subsection{Individual Procedures}

With regard to individual procedures, ideally the following staff roles are present for each case:

- One first operator: a neurointerventionist

- One assistant: a second scrubbed individual (ie, a supporting AIS interventionist, physician in training (resident or fellow), nurse practitioner, physician assistant, a scrub nurse, or a radiographer)

- One radiographer

- One nurse or nurse assistant

- Regardless of the type of anesthesia, an anesthesiology service must be readily available $24 / 7$.

As a minimum, a neurointerventionist, a radiographer, and appropriately trained nursing must be present.

\subsection{Quality Improvement Processes}

Treatment of AIS by using AISI techniques is a novel method that involves the consumption of significant human and material resources and carries the risk of severe complications. Accurate documentation of medical and technical details as well as patient outcome and follow-up results is inevitable to ensure the highest benefit of such complex and demanding procedures.

To secure such documentation and data management, it is recommended that:

- The level 2 stroke center team includes a dedicated individual, preferably a stroke nurse or a stroke fellow, with the responsibility of data recording and data base management.

- All technical and clinical data of AISI procedures, patient outcomes, and follow-up must be entered into an electronic data base either locally or (preferably) nationally or internationally.

- The center shall establish target time metrics for all cases in accordance with the most recent requirements by international standards. Cases that exceed their chosen metrics should trigger an internal process for quality improvement. ${ }^{17}$

- The database should be regularly audited. At a minimum, process metrics such as time from arrival to intravenous tissue plasminogen activator, to start of angiography, and to recanalization, as well as overall recanalization rates, are to be reviewed and compared against reasonable published benchmarks.

AJNR Am J Neuroradiol 39:E112-E117 Nov 2018 www.ajnr.org E115 
- The center provides routine continuing education (suggested minimum of 8 hours per year) related to cerebrovascular disease and stroke for all core members of the center, as designated by the medical director.

- All cases of symptomatic intracranial hemorrhage shall be reviewed. For the purposes of this document, we broadly define symptomatic intracranial hemorrhage as the presence of new intracranial hemorrhage on post-treatment brain imaging, with clinical deterioration that is potentially attributable to the hemorrhage.

- Standardized care pathways should be implemented with clinical practice guidelines, order sets, and other tools to ensure consistent care delivery and minimize practice variability. This should apply to providers, and nursing and ancillary staff. These pathways should be developed by the multidisciplinary Asian-Australian Federation of Interventional and Therapeutic Neuroradiology (AAFITN), Australianand New Zealand Society of Neuroradiology (ANZSNR), American Society of Neuroradiology (ASNR), Canadian Society of Neuroradiology (CSNR), European Society of Minimally Invasive Neurologic Therapy (ESMINT), European Society of Neuroradiology (ESNR), European Stroke Organization (ESO), Japanese Society for NeuroEndovascular Therapy (JSNET), The French Society of Neuroradiology (SFNR) Ibero-Latin American Society of Diagnostic and Therapeutic Neuroradiology (SILAN), Society of NeuroInterventional Surgery (SNIS), Society of Vascular and Interventional Neurology (SVIN), World Stroke Organization (WSO), World Federation of Interventional Neuroradiology (WFITN) leadership of the center and reflect evidence based practice.

\section{Community and EMS Outreach}

Outstanding stroke care starts not in the hospital but in the field. Increasingly, operators will likely promote selection of the most appropriate destination for suspected ELVO patients based on distance to a center from the field. ${ }^{19}$ Such a mechanism should decrease time to treatment. As such, the level 2 center should interface with local emergency medical services (EMS) in order to coordinate care in the prehospital arena.

Specifically, we feel there are some key items in this area.

- Representatives of the center shall work with local and regional EMS officials to ensure they are aware of the system's capabilities, as well as which patients (based on the region's chosen severity scale) are appropriate for direct field triage to the level 2 or 1 centers.

- Additionally, some patients may be distant from the level two (or 1 ) and present to a level 3 center. The level 2 center should work with these local centers to assist in identification of suspected or confirmed ELVO patients and facilitate rapid transfer as part of a 'hub and spoke' model of care. However, if a level 1 center is available in a similar transfer time, it is preferable that interfacility transfers are directed to the highest level facility.

- A mechanism should exist for providing feedback to the EMS and referring non-thrombectomy centers to highlight which aspects of care went well and identify areas for improvement. This would be similar to quality assessment work done on patients presenting directly to the level 2 and 1 centers.

\section{AUTHOR AFFILIATIONS}

'Hôpital Maison-Blanche, Université Reims-Champagne-Ardenne (URCA), Reims, Frane; ${ }^{2}$ Warren Alpert School of Medicine at Brown University, Providence, Rhode Island, USA; ${ }^{3}$ National Institute of Clinical Neurosciences, Budapest, Hungary; ${ }^{4}$ Massachusetts General Hospital, Harvard Medical School, Boston, Massachusetts, USA; ${ }^{5}$ Erlanger Medical Center, Chattanooga, Tennessee, USA; ${ }^{6}$ Neuroendovascular Therapy Centre, Aichi Medical University, Aichi-gun, Japan; ${ }^{7}$ Pantai Hospital, Kuala Lumpur, Malaysia; ${ }^{8}$ Monash University, Clayton, Victoria, Australia; ${ }^{9}$ The University of Melbourne, The Royal Hospital of Melbourne, Parkville, Victoria, Australia; ${ }^{10}$ University of Queensland, Royal Brisbane and Women's Hospital, Brisbane, Queensland, Australia; ${ }^{11}$ University of Wisconsin, Madison, Wisconsin, USA; ${ }^{12}$ Northwell Health Donald and Barbara Zucker School of Medicine, New York, New York, USA; ${ }^{13}$ Queen's University, Kingston, Ontario, Canada; ${ }^{14}$ Karolinska University Hospital, Stockholm, Sweden; ${ }^{15}$ University Medical Centre Hamburg-Eppendorf, Hamburg, Germany; ${ }^{16}$ Istanbul University, Cerrahpasa Medical School, Istanbul, Turkey; ${ }^{17}$ Hospital Garcia de Orta, Hospital da Luz, Lisbon, Portugal; ${ }^{18}$ Hospital Univesitari Vall d'Hebron, Barcelona, Spain; ${ }^{19}$ Neurology, Inselspital, University Hospital Bern and University of Bern, Bern, Switzerland; ${ }^{20}$ University of Perugia, Perugia, Italy; ${ }^{21}$ University Medical Centre, Utrecht, The Netherlands; ${ }^{22}$ Kobe City Medical Centre General Hospital, Kobe, Japan; ${ }^{23}$ University of Tsukuba, Tsukuba, Japan; ${ }^{24}$ Hyogo College of Medicine, Nishinomiya, Japan; ${ }^{25} \mathrm{CHU}$ Nantes, Nantes, France; ${ }^{26}$ Hospital Universitário Santa Maria, Lisbon, Portugal; ${ }^{27}$ Hospital Clinico Universitário, Santiago de Compostela, Espana; ${ }^{28}$ The Methodist Hospital, Houston, Texas, USA; ${ }^{29}$ University of Kentucky, Lexington, Kentucky, USA; ${ }^{30}$ Herbert Wertheim College of Medicine, Florida International University, Miami Cardiac and Vascular Institute, Baptist Hospital, Miami, Florida, USA; ${ }^{31}$ UCLA, Los Angeles, California, USA; ${ }^{32}$ Marcus Stroke and Neuroscience Center, Grady Memorial Hospital, Emory University School of Medicine, Atlanta, Georgia, USA; ${ }^{33}$ University of Heidelberg, Heidelberg, Germany; ${ }^{34}$ Danube University Krems, Krems, Austria; ${ }^{35}$ Melbourne Brain Centre at Royal Melbourne Hospital, University of Melbourne, Parkville, Victoria, Australia; ${ }^{36}$ Groote Schuur Hospital, University of Cape Town, Cape Town, South Africa; ${ }^{37}$ Ramathibodi Hospital, Mahidol University, Bangkok, Thailand; ${ }^{38}$ Kameda Medical Center, Kamagowa City, Chiba, Japan; ${ }^{39}$ University of Toronto, Toronto Western Hospital, Toronto, Ontario, Canada

\section{REFERENCES}

1. Anon. Training guidelines for endovascular ischemic stroke intervention: an international multi-society consensus document. J Neurointerv Surg 2016;8:989-91

2. Leslie-Mazwi T, Chandra RV, Baxter BW, et al. Society of NeuroInterventional Surgery. ELVO: an operational definition. J Neurointerv Surg 2018;10:507-9

3. Lima FO, Furie KL, Silva GS, et al. Prognosis of untreated strokes due to anterior circulation proximal intracranial arterial occlusions detected by use of computed tomography angiography. JAMA Neurol 2014;71:151-7

4. Saver JL, Goyal M, Bonafe A, et al. Stent-retriever thrombectomy after intravenous t-PA vs. t-PA alone in stroke. $N$ Engl $\mathrm{J} \mathrm{Med}$ 2015;372:2285-95

5. Jovin TG, Chamorro A, Cobo E, et al. Thrombectomy within 8 hours after symptom onset in ischemic stroke. $N$ Engl J Med 2015;372:2296-306

6. Goyal M, Demchuk AM, Menon BK, et al. Randomized assessment of rapid endovascular treatment of ischemic stroke. $N$ Engl J Med 2015;372:1019-30

7. Campbell BC, Mitchell PJ, Kleinig TJ, et al. Endovascular therapy for ischemic stroke with perfusion-imaging selection. $N$ Engl J Med 2015;372:1009-18

8. Berkhemer OA, Fransen PS, Beumer D, et al. A randomized trial of intraarterial treatment for acute ischemic stroke. $N$ Engl J Med 2015;372:11-20

9. Goyal M, Menon BK, van Zwam WH, et al. Endovascular thrombectomy after large-vessel ischaemic stroke: a meta-analysis of individual patient data from five randomised trials. Lancet 2016;387:1723-31

10. Saver JL, Goyal M, van der Lugt A, et al. Time to treatment with endovascular thrombectomy and outcomes from ischemic stroke: a meta-analysis. JAMA 2016;316:1279-88

11. Mocco J, Zaidat OO, von Kummer R, et al. Aspiration thrombec- 
tomy after intravenous alteplase versus intravenous alteplase alone. Stroke 2016;47:2331-8

12. Bracard S, Ducrocq X, Mas JL, et al. Mechanical thrombectomy after intravenous alteplase versus alteplase alone after stroke (THRACE): a randomised controlled trial. Lancet Neurol 2016;15:1138-47

13. Nogueira RG, Jadhav AP, Haussen DC, et al. Thrombectomy 6 to 24 hours after stroke with a mismatch between deficit and infarct. N Engl J Med 2018;378:11-21

14. Albers GW, Marks MP, Kemp S, et al. Thrombectomy for stroke at 6 to 16 hours with selection by perfusion imaging. $N$ Engl J Med 2018;378:708-18

15. Rinaldo L, Brinjikji W, Rabinstein AA. Transfer to high-volume centers associated with reduced mortality after endovascular treatment of acute stroke. Stroke 2017;48:1316-21

16. Jansen O, Szikora I, Causin F, et al. Standards of practice in interventional neuroradiology. Neuroradiology 2017;59:541-4
17. Harold JG, Bass TA, Bashore TM, et al. ACCF/AHA/SCAI 2013 update of the clinical competence statement on coronary artery interventional procedures: a report of the American College of Cardiology Foundation/American Heart Association/American College of Physicians Task Force on Clinical Competence and Training (writing committee to revise the 2007 clinical competence statement on cardiac interventional procedures). Circulation 2013;128:436-72

18. McTaggart RA, Ansari SA, Goyal M, et al. Initial hospital management of patients with emergent large vessel occlusion (ELVO): report of the standards and guidelines committee of the Society of NeuroInterventional Surgery. J Neurointerv Surg 2017;9:316-23

19. Zhao H, Coote S, Pesavento L, et al. Large vessel occlusion scales increase delivery to endovascular centers without excessive harm from misclassifications. Stroke 2017;48:568-73 